\title{
Spyglass ${ }^{\circledR}$ na resolução de coledocolitíase primária: um relato de caso
}

\author{
Fernanda Barros Viana ${ }^{1}$ \\ Flavio Hayato Ejima \\ Lígia Aparecida Machado ${ }^{3}$ \\ Anna Paula Mendanha da Silva Aureliano ${ }^{1}$ \\ Liliana Sampaio Costa Mendes ${ }^{4}$
}

\author{
${ }^{1}$ Médica Residente do Programa de Gastroenterologia do Hospital de Base do Distrito Federal/Instituto de Gestão \\ Estratégica do Distrito Federal. \\ ${ }^{2}$ Médico Gastroenterologista e Endoscopista do HBDF/IGESDF. \\ ${ }^{3}$ Técnica de Enfermagem do HBDF/IGESDF. \\ ${ }^{4}$ Médica Gastroenterologista, Doutora em Hepatologia pela Universidade de São Paulo, assistente do Departamento \\ de Hepatologia do HBDF/IGESDF.
}

\section{RESUMO}

Introdução: A observação endoscópica direta das vias biliopancreáticas tem sido aprimorada durante décadas visando maior efetividade diagnóstica e terapêutica. Em 2006 foi desenvolvida a colangioscopia de operador único (SPYGLASS ${ }^{\circledR}$ ), que permite a tripsia de cálculos grandes, bem como tratamento de estenoses e biópsias de tumores das vias biliares. Relato de Caso: Apresentamos um caso de coledocolitíase primária por cálculo gigante extraído após litotripsia a laser com a técnica da colangioscopia peroral por operador único. Discussão: Antigamente a escolha para pacientes com coledocolitíase por cálculo maior que $1,5 \mathrm{~cm}$ era a derivação biliodigestiva. Hoje a colangioscopia representa um importante avanço na endoscopia terapêutica devido à alta acurácia e precisão na manipulação da árvore biliar, evitando realização de cirurgias de grande porte. Conclusão: Pode-se concluir que, apesar do alto custo, a colangioscopia representa uma opção endoscópica inovadora, acurada e pouco invasiva, capaz de evitar ato cirúrgico de grande porte, além de estar bem indicada para biópsia em vias biliares e tratamento de pacientes com estenose em via biliar e cálculos gigantes.

Palavras-chave: coledocolitíase, via biliar, cálculos biliares, endoscopia

Spyglass ${ }^{\circledR}$ in the resolution of primary choledocolithiasis: a case report

\begin{abstract}
Introduction: Direct endoscopic observation of biliopancreatic pathways has been improved for decades, aiming at greater diagnostic and therapeutic effectiveness. In 2006, single-operator cholangioscopy (SPYGLASS $®$ ) was developed, which allows for large-stone trypsia, as well as treatment of biliary tumor tumors and strictures. Case Report: We present a case of primary choledocholithiasis by giant stone extracted after laser lithotripsy with the technique of peroral cholangioscopy by a single operator. Discussion: In the past, the choice for patients with choledocholithiasis by calculation greater than $1.5 \mathrm{~cm}$ was the biliodigestive derivation. Today cholangioscopy represents an important advance in therapeutic endoscopy due to the high accuracy and precision in the manipulation of the biliary tree, preventing a major surgery to be performed. Conclusion: From this case report, it can be concluded that, despite the high cost, cholangioscopy represents an innovative, accurate and low-invasive endoscopic option,
\end{abstract}


capable of preventing major surgery, being well suited for biliary biopsy and treatment of patients with biliary stenosis and giant stones.

\section{Keywords: Choledocholithiasis, bile ducts, gallstones, endoscopy}

\section{INTRODUÇÃO}

A presença de cálculos na via biliar abrange até $20 \%$ da população europeia ${ }^{(1)}$. Nos Estados Unidos os dados chegam a 20 milhões de pessoas afetadas ${ }^{(2)}$. No Brasil, 9,3\% da população com mais de 20 anos apresenta cálculos na vesícula (colecistolitíase) e acima de 65 anos essa prevalência chega a 30\% ${ }^{(3-4)}$.

Há algumas complicações possíveis para os cálculos de vias biliares. A impactação do cálculo no infundíbulo da vesícula biliar ou no ducto cístico causa a distensão da vesícula biliar e a contração da mesma, o que gera uma dor característica em hipocôndrio direito, a cólica biliar. Se o cálculo não migrar e desobstruir a vesícula, a inflamação dela leva à colecistite aguda ${ }^{(4)}$.

O cálculo formado na vesícula pode impactar ainda no colédoco, gerando a coledocolitíase. Se houver a infecção das vias biliares pela deficiência na drenagem pelo colédoco obstruído, teremos um quadro de colangite ${ }^{(4)}$. Se o cálculo migrar e ficar impactado na região da válvula íleo-cecal temos um quadro obstrutivo denominado íleo biliar ${ }^{(5)}$. A pancreatite aguda é também uma complicação da doença litiásica, quando há obstrução do ducto pancreático ${ }^{(4)}$.

O cálculo neoformado no colédoco (coledocolitíase primária) ocorre em pacientes com estase biliar (fibrose cística, estenose de colédoco póscolecistectomia, papilites estenosantes), idosos com ducto biliar dilatado e divertículo ampular e em infecções recorrentes ou persistentes da via biliar, como em alguns países asiáticos ${ }^{(1,4)}$.

Durante décadas tem sido aprimorada a técnica de diagnóstico e terapêutica das vias biliares. Na década de 60 surgiu a colangiografia retrógrada endoscópica, um 
método que permitia o estudo das vias biliares por via endoscópica. As desvantagens eram a imagem por fluoroscopia, a limitação na retirada de cálculos maiores que $15 \mathrm{~mm}$ e no diagnóstico de tumores de vias biliares ${ }^{(6)}$.

Em 1975 surgiu a colangioscopia com visualização direta por duplo operador: "mother-daughter" (duodenoscopista/ colangioscopista). As principais limitações eram necessitar de dois endoscopistas experientes, a baixa qualidade da câmera de fibra óptica e a impossibilidade de irrigar a via biliar e de defletir a ponta ${ }^{(7)}$.

Em 2006 foi descrita a colangioscopia por operador único, o SPYGLASS ${ }^{\circledR}{ }^{(6)}$. Em 2009 foi aprovado pelo FDA (Food and Drug Administration), nos Estados Unidos, e em 2014 foi publicada a primeira série brasileira, com 20 casos, feita pela Universidade de São Paulo - USP ${ }^{(8)}$.

As funções do SPYGLASS ${ }^{\circledR}$ incluem biópsia de lesões intrínsecas e extrínsecas da via biliar, litotripsia de cálculos grandes, diagnóstico de estenoses biliares, estudo de pacientes com hemobilia, colocação de prótese no ducto cístico ${ }^{(6,9) \text {. }}$

A litotripsia pelo SPYGLASS ${ }^{\circledR}$ pode ser de duas formas: eletrohidráulica ou a laser. A forma eletrohidráulica inclui a infusão de solução salina com alta pressão e a administração do choque pelo colangioscópio. A técnica a laser consiste na transmissão deste por fibra de quartzo. Apesar de mais caro e consistir em um material mais frágil, é mais eficiente ${ }^{(6)}$.

Apresentamos um caso bem sucedido de coledocolitíase primária com cálculo gigante (maior que $15 \mathrm{~mm}$ ) em uma paciente colecistectomizada há 20 anos, cujos cálculos não conseguiram ser extraídos por CPRE convencional, necessitando do SPYGLASS $^{\circledR}$ com litotripsia a laser. O estudo do caso foi realizado após a aprovação no comitê de ética em pesquisa (CAAE 08261419.7.0000.8153) e preenchimento do termo de consentimento livre e esclarecido. 


\section{RELATO DO CASO}

Paciente de 63 anos, do sexo feminino, hipertensa, dislipidêmica, com obesidade grau 1 e já submetida a colecistectomia em 1997, apresentou quadro de pancreatite biliar e coledocolitíase em 2017. Foi submetida a exploração da via biliar para confecção de derivação biliodigestiva, uma vez que a colangiografia retrógrada endoscópica (CPRE) não estava disponível. No intraoperatório foram vistas alterações no peritônio, as quais poderiam sugerir carcinomatose periotoneal, de forma que o cirurgião optou por fazer biópsias e suspender o procedimento previsto. Posteriormente as biópsias vieram negativas para malignidade.

Em fevereiro de 2018 a paciente apresentou novo episódio de coledocolitíase, sendo então submetida a CPRE. Verificaram durante o procedimento a dilatação das vias biliares intra e extra-hepáticas, com múltiplos cálculos de colédoco com cerca de 1,6 cm. Foi realizada papilotomia, com dilatação da papila maior com balão de $15 \mathrm{~mm}$, retirado um cálculo com balão extrator e colocada prótese em colédoco, não sendo possível retirar os demais cálculos.

Como não foi possível retirar os cálculos grandes, foi agendada CPRE com litotripsia a laser por SPYGLASS ${ }^{\circledR}$. O procedimento aconteceu sem intercorrências, com retirada dos cálculos após fragmentação dos mesmos.

A paciente evoluiu assintomática após um ano de acompanhamento ambulatorial. 


\section{DISCUSSÃO}

Anteriormente os pacientes com cálculos gigantes (maiores que $15 \mathrm{~mm}$ ) na via biliar eram submetidos à cirurgia desobstrutiva com derivação da via biliar hepaticojejunostomia em Y de Roux.

Atualmente, com a técnica da colangioscopia por operador único é possível realizar a litotripsia dos cálculos, seja ela a laser ou por eletrohidráulica, de forma endoscópica. Diversas são as vantagens visto ser uma técnica bem menos invasiva, com menor resposta metabólica ao trauma, menor tempo de internação intra hospitalar e melhor recuperação.

As possibilidades do $\mathrm{SPYGLASS}^{\circledR}$ não se resumem à quebra dos cálculos intraductais, uma vez que a realização de biópsias de tumores dentro da via biliar e a colocação de próteses também são possíveis.

Apesar de ter sido descrita em 2006, ainda é uma realidade para poucos pacientes pelo alto custo e pela necessidade de profissional experiente.

A partir deste relato de caso fica claro que o SPYGLASS ${ }^{\circledR}$ representa progresso considerável em relação à tecnologia endoscópica, apesar do alto custo, visto a alta acurácia e precisão na visualização direta e manipulação da árvore biliar, evitando a realização de cirurgia de grande porte. Além disso, a colangioscopia é bem indicada para tratamento de pacientes com cálculos gigantes e estenose em via biliar, além de poder realizar biópsias em vias biliares.

\section{CONCLUSÃO}

O SPYGLASS ${ }^{\circledR}$ é uma modalidade terapêutica eficiente e tem menor morbidade do que a cirurgia na abordagem de cálculos grandes. 


\section{REFERÊNCIAS}

1. EASL Clinical Practice Guidelines on the prevention, diagnosis and treatment of gallstones. J.Hepatol (2016). Disponível em: http:// dx.doi.org/10.1016/j.jhep.2016.03.005.

2. Everhart JE, et al. Prevalence and ethnic differences in gallbladder disease in the United States, Gastroenterology. 1999;117(3):632.

3. Coelho JC, et al. Prevalence of gallstones in a Brazilian population, Int Surg., 1999, Jan-Mar;84(1):25-8.

4. Sipahi HM, et al. Calculose biliar. In: Schlioma Z, Jaime NE (eds). Tratado de gastroenterologia: da graduação à pós-graduação -- 2. ed. -- São Paulo: Editora Atheneu, 2016. p1013 - 1023.

5. Guimarães S., et al. Íleo biliar - uma complicação da doença calculosa da vesícula biliar, Rev. Bras. Geriatr. Gerontol., Rio de Janeiro, 2010; 13(1):159-163.

6. Pereira P, et al. Peroral Cholangiopancreatoscopy with the SPYGLASS®® System: What do we Know 10 Years Later, J Gastrointestin Liver Dis, June 2017 Vol. 26 No 2: 165-170.

7. Itoi T, Cholangioscopy, In: S. Jonnalagadda (ed.). Gastrointestinal endoscopy. Springer Science Business Media New York 2015. p23-37.

8. Moura EGH, et al. Cholangioscopy in bile duct disease: a case series. Arq Gastroenterol, [Internet]. 2014 Sep [cited 2019 Dec 03] ; 51(3):250254.Disponível

em: 
http://www.scielo.br/scielo.php?script=sci_arttext\&pid=S000428032014000300250\&lng=en.

9. Navaneethan U, et al. Single-operator cholangioscopy and targeted biopsies in the diagnosis of indeterminate biliary strictures: a systematic review. Gastrointestinal Endoscopy. Gastrointest Endosc. 2015 Oct;82(4):60814.e2. 